itrary function of $t$, the same solution will represent the gravitational field of a radiating star found by $u^{2}$, thus showing that our solution of a non-static mass is the natural generalization of Schwarzschild's solution for a static mass.

Again, for describing the field of a mixture of matter and flowing radiation within the interior of a star, where the energy tensor is of the form ${ }^{3}$ :

$$
\begin{array}{r}
T_{\mu \nu}=(p+\rho) v_{\mu} v_{\nu}-p g_{\mu \nu}+\sigma w_{\mu} w_{\nu}, \\
v_{\mu} v^{\mu}=1, w_{\mu} w^{\mu}=0,
\end{array}
$$

we can choose co-moving Newtonian co-ordinates in which $v^{1}=v^{2}=v^{3}=0, w^{2}=w^{3}=w^{4}=0$. $\quad \mathrm{We}$ shall then find that:

$$
T_{1}{ }^{1}=T_{2}{ }^{2}=T_{3}{ }^{3}=-p ; T_{4}{ }^{4}=\rho .
$$

These relations are the same as the familiar relations for static solutions. Of course, here, $T_{1}{ }^{4} \neq 0$.

Recently, we have found that even in the unified field theories described by a non-symmetrical $g_{i k}$, co-ordinates of this type introduce a great deal of simplification in problems involving radiation flow. Details of this investigation are awaiting publication elsewhere.

Vallabh Vidyanagar, Anand, India. July 26.

* Springer Research Scholar, University of Bombay

'Eisenhart, I. P., "Riemannian Geometry", Appendix 25 (Princeton, 1949)

Vaidya, P. C., Proc. Ind. Acad. Sci., A, 33, 264 (1951).

saidya, P. C., Phys. Rev., 83, 10 (1951).

\section{Serological Relationships of Hyrax and Elephant}

OrIGINally classed with the Pachyderma, the hyraxes (rock rabbits or conies) have in turn been placed in the orders Rodentia and Perissodactyla. More recently, they have been placed in a separate order, the Hyracoidea ${ }^{1}$. It is, however, still open to discussion whether there is not a relation between hyrax and elephant; one of the more recent contributions on the subject is based on the similarity of the placental formation in animals of the two orders concerned ${ }^{2}$.

In the course of some recent work on the development of the use of precipitating antisera for the identification of blood meals of tsetse flies and other African blood-sucking arthropods, an antigenic relationship between elephant and hyrax has been observed. Serological relationships have been noted by various workers between related animals of many different species since the original work of Nuttall ${ }^{3}$ on this subject. Qualitative determinations made by the ring test have been shown to be valid, providing the antisera used in the reaction are devoid of non-specific antibodies. Before absorption, antisera prepared in rabbits by repeated injections of alumprecipitated serum give reactions at a high titre with the serum of all the mammals which were tested and which included representative samples from most orders ${ }^{4}$.

'Two antisera were prepared in rabbits by the repeated injections of alum-precipitated sera of the Indian elephant (Elephas elephas) and hyrax (Heterohyrax syriacus prittwitzi Brauer) respectively, and both antisera gave positive reactions at high titre with the sera of all mammals which were tested. Each antiserum was then absorbed with the sera of man, ox, sheep, horse, pig, dog and cat in the pro- portions necessary to remove the corresponding antibodies. These absorbed antisera were then re-tested as before with the sera of a wide range of species of mammals and were found to react only with elephant and hyrax in both cases. The anti-elephant serum reacted at a titre of $1: 64,000$ with elephant serum and at $1: 32,000$ with hyrax serum, while the anti-hyrax serum reacted at a titre of $1: 16,000$ with hyrax serum and at $1: 8,000$ with elephant serum.

These observations, which were similar to those previously made with known related mammals, indicate a very marked relationship between the sera of hyrax and elephant; they are also of interest in relation to the taxonomic position of the Hyracoidea.

I am grateful to the Zoological Society of London for supplying the elephant serum, and to T. C.S. Morrison Scott for identifying the species of hyrax.

Bernard WeITz

Lister Institute of Preventive Medicine,

Elstree, Herts. May 22.

${ }^{2}$ Simpson, G. G., Bull. Amer. Mus. Nat. Hist., 85, 250 (1945),

2 Wislocki, G. B., and van der Westhuysen, O. P., Contrib. Embryol. Carneg. Inst., 28, 171 (1940).

${ }^{3}$ Nuttall, G. W. W., "Blood Immunity and Blood Relationshins" (Camb. Univ. Press, 1904).

‘ Weitz, B., J. Hyg., Camb., 50, 3, 275 (1952).

\section{Assessment of Consciousness during Curarization}

MODERN surgical techniques and experimental methods make increasing use of muscular relaxants combined with anæsthetics to produce the optimum conditions for surgery. This practice leads to certain difficulties in assessing consciousness, since the usual muscle reflexes are impaired by the action of the relaxant. The change in heart-rate in response to a pain stimulus has recently been investigated and found to be a reliable indicator of consciousness during electrical curarization in animals, and during ether anæsthesia in man!. It therefore seemed possible that this cardiac pain reflex might be useful as an index of consciousness when muscular relaxants were being used.

'Flaxedil' was injected intravenously into rabbits so that the voluntary muscles were paralysed, and the heart-rate was recorded continuously on one channel of an electro-encephalograph machine; the pain stimulus consisted of a pin-prick on the nose of the animal. This stimulus resulted in an increase in the heart-rate which varied from 9 to 14 per cent with different individuals and lasted for about thirty seconds ; the cardiac pain reflex before injection was similar in size and duration, but during light anæsthesia it was reduced, and in surgical anæsthesia it was abolished.

The pupillary reflex to light, which can sometimes be used when voluntary muscle is paralysed, is not, easy to observe after injection of 'Flaxedil' because ptosis and nystagmus occur. If a suitable heart-rate indicator is available, or could be designed, it might now have considerable practical application in human and veterinary surgery when curarizing drugs or new anæsthetics are being used.

Phyllis G. Croft

Neuropsychiatric Research Centre,

Whitchurch Hospital,

Cardiff.

Sept. 1.

${ }^{3}$ Croft, P. G., J. Ment. Sci., 98, 421, 427 (1952). 